\title{
PRODUCTOS ESTRATEGICOS EN SALUD: UNA NECESIDAD PARA AFRONTAR ADECUADA Y OPORTUNAMENTE LAS PANDEMIAS Y EPIDEMIAS
}

\section{STRATEGIC HEALTH PRODUCTS: A NECESSITY TO ADEQUATE AND TIMELY ADDRESS PANDEMICS AND EPIDEMICS}

\author{
César Cabezas (iD) 1,2,a \\ Instituto Nacional de Salud. Lima, Perú. \\ Facultad de Medicina, Universidad Nacional Mayor de San Marcos \\ Médico infectólogo-Tropicalista
}

El virus SARS-CoV-2 causante de la pandemia del COVID-19 con su gran eficiencia de transmisión de persona a persona, sus permanentes variantes y su nivel relativamente alto de morbilidad y mortalidad, especialmente entre personas mayores y aquellos con comorbilidad subyacente, ha trastornado nuestro planeta en tiempos bastante cortos, consiguiendo bloquear las principales actividades humanas. Según la Organización Mundial de la Salud (OMS) ${ }^{(1)}$ se han reportado en el mundo 239,437,517 casos confirmados de COVID-19, incluyendo 4,879,235 decesos, mientras que en el Perú tenemos 2,187,368 casos confirmados y 199,775 fallecidos por esta causa reportados por el Ministerio de Salud ${ }^{(2)}$ hasta la fecha de redactar este editorial y con respecto a las muertes prematuras se calcula que el COVID-19 ha inducido la pérdida de más de 20,5 millones de años de vida en todo el mundo, tres cuartas partes de los años de vida perdidos (AVP) resultan de muertes en menores de 75 años, casi un tercio de muertes en menores de 55 años; y los hombres han perdido un $45 \%$ más de años de vida que las mujeres ${ }^{(3)}$.

Sin embargo, pandemias como el COVID-19 no son fenómenos nuevos, las enfermedades infecciosas emergentes siempre se han dado y amenazado a los seres humanos casi desde el neolítico, cuando el ser humano empieza a manipular de manera sistemática y generalizada la naturaleza. Emergieron enfermedades como la viruela, malaria, sarampión, la peste bubónica que mató millones de personas en Asia y Europa. En 1918 la pandemia de influenza mató a más de 50 millones personas, la pandemia del VIH/SIDA, identificada en 1981, ha matado al menos 37 millones de personas. La última década ha sido testigo de explosiones pandémicas: influenza "porcina" H1N1 (2009), dengue, chikungunya (2014) y Zika (2015), así como emergencias de tipo pandémico como la fiebre del Ébola en gran parte de África (2014). El síndrome respiratorio agudo severo (SARS) coronavirus (SARS-CoV) surgió de un huésped animal, entre el 2002-2003, para causar una casi pandemia antes de desaparecer probablemente en respuesta a las medidas de control de salud pública. Los coronavirus relacionados con el síndrome respiratorio de Oriente Medio (MERS) (MERS-CoV) surgió en humanos a partir de camellos y dromedarios en 2012, pero desde entonces se ha transmitido de manera ineficiente entre humanos. El COVID-19, reconocido a finales de 2019, es el último y el mejor ejemplo de una pandemia inesperada, novedosa y devastadora enfermedad ${ }^{(4)} y$ con esta experiencia, aun en curso, se puede concluir que hemos entrado en una era pandémica ${ }^{(5)}$.

El Perú y los países de las Américas no son ajenos a estos embates de enfermedades in-

Citar como: Cabezas C. Productos estratégicos en salud: una necesidad para afrontar adecuada y oportunamente las pandemias y epidemias. Rev Peru Med Exp Salud Publica. 2021;38(3):377-80. doi: https://doi.org/10.17843/ rpmesp.2021.383.9776. fecciosas emergentes que se suman a los que ya tenemos en diferentes regiones en particular en nuestro país tan megadiverso, con nichos ecológicos diferenciados que hacen posible la aparición reaparición o mantenimiento de enfermedades infecciosas. En la figura 1 podemos mostrar la emergencia o reemergencia de enfermedades a través de los años. Además de las enfermedades mencionadas anteriormente, debemos destacar enfermedades como la rabia canina y silvestre, fiebre de mayaro, oropouche, dengue, fiebre amarilla, zika, chikungunya, estas cuatro últimas transmitidas por el Aedes aegypti que es un vector que evidencia el problema de la disponibilidad y almacenamiento de agua en nuestras poblaciones y en el 
Dengue 1, 2 (1990-21)

Chikungunya (2015)

Malaria P. falciparum 2011

Rickettsias
Peste (1994)

Sarampión 2016

Cholera (1991)
Encefalitis Equina Venezolana

(1993-95)

Ricketsiosis (2008)

Leptospirosis (1998)

Oropuche (1992)

Malaria $P$. falciparum

(1994-97)

Mayaro (1995)

Rabia (1990-97)

Tifus (1990-98)

Oropouche

(1994)

Bartonellosis

Fiebre Amarilla

COVID-19 (2020):

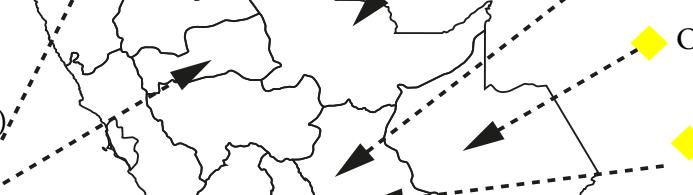

Barton

(1994)

HIV / AIDS

Influenza AH 2009...

Infecciones

respiratorias

Rabia canina

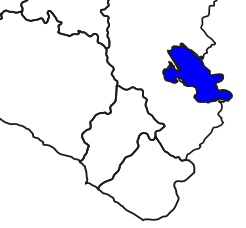

Figura 1. Enfermedades infecciosas emergentes y reemergentes en Perú.

caso de la fiebre amarilla; si bien, actualmente tenemos la forma selvática, siempre está como una espada de Damocles, el riesgo de su reurbanización por la presencia del mosquito Aedes aegypti en ciudades de la costa y amazonia, más aun considerando lo ocurrido en el Brasil el 2017 donde se presentó una epidemia de fiebre amarilla periurbana, y Brasil aun siendo productor de la vacuna antiamarílica tuvo que racionalizar su uso diluyendo en cinco la dosis habitual de este biológico ${ }^{(6)}$.

Todo lo anterior nos conlleva a pensar en cómo vigilar estas enfermedades, prevenirlas y controlarlas, entonces surge la necesidad de un sistema de salud pública sólido que abarque simultáneamente la salud colectiva que tiene que ver con las intervenciones en poblaciones y el componente de salud individual que tiene que ver con el fortalecimiento de los servicios de salud para la atención de los pacientes desde el primer nivel de atención. Desde Alma Ata en 1978 se declaraba enfrentar las profundas inequidades económicas y sociales, y espera que de ser un discurso pase a ser una realidad concreta. Estas necesidades se han evidenciado en la pandemia que aun asistimos. En ese contexto debemos contar con desarrollos tecnológicos para el diagnóstico oportuno, recursos terapéuticos y vacunas.
En situaciones similares, cuando hubo el acuerdo mundial de la eliminación de la viruela, la única enfermedad erradicada en el mundo, los Institutos Nacionales de Salud jugaron un rol importante, como fue la producción de vacuna antivariólica liofilizada con las que el Perú contribuyó a su erradicación, habiendo en su momento exportado estas vacunas a Ecuador, Bolivia e incluso Canadá.

La crisis sanitaria por la COVID-19 evidencióla dependencia delasimportacionesdemedicamentosyotrastecnologíassanitarias, la vulnerabilidad de las cadenas de suministro en los contextos de emergencias, y la alta heterogeneidad de las Américas en términos de sus capacidades de investigación, desarrollo y producción de vacunas para la COVID-19. El aumento inesperado, sincrónico y acelerado en la demanda de ciertos dispositivos médicos, conllevó a situaciones de desabastecimiento e incrementos desmedidos de precios ${ }^{(7)}$ como lo ocurrido en la primera y segunda ola en el Perú, en la que se entrecruzaban la ciencia la tecnología y el negocio, registrándose también el desabastecimientos de otras tecnologías sanitarias, como los ventiladores mecánicos, la producción de oxígeno, oxímetros y algunos medicamentos utilizados en las unidades de cuidados intensivos.

América Latina y el Caribe tienen una alta dependencia de materias primas y medicamentos y otras tecnologías 
sanitarias que son importadas, de tal modo que la Comisión Económica para América Latina y el Caribe (CEPAL) de la Organización de las Naciones Unidas estimó que en el año 2018 solo el 4\% de las importaciones de América Latina y el Caribe de un conjunto estratégico de tecnologías sanitarias tenían como origen la propia subregión ${ }^{(8)}$. De modo que el enfrentamiento a la pandemia del COVID-19 se dio con muchas limitaciones de disponibilidad y oportunidad, con las consecuencias que ello ha significado en morbilidad y mortalidad.

$\mathrm{Al}$ inicio y en el curso de la pandemia se dieron iniciativas de la OMS, en conjunto con la Alianza para las Vacunas (GAVI), y la Coalición para la Promoción de Innovaciones en pro de la Preparación ante Epidemias (CEPI) del mecanismo para el acceso mundial a las vacunas contra la COVID-19 (COVAX ${ }^{\left({ }^{9}\right)}$. Sin embargo, no se ha podido cumplir con abastecer equitativamente con vacunas a todos los países sobre todo a los que están en vías de desarrollo. Entre otras iniciativas en la Región de las Américas, se registran proyectos en Argentina, Brasil, Canadá, Cuba, Estados Unidos y México, algunas de las cuales ya han alcanzado autorización de los organismos reguladores igualmente tanto países desarrollados como países en desarrollo han participado en la realización de diversos ensayos clínicos de medicamentos y otras tecnologías sanitarias en las América (OMS), pero, y pese al gran valor de tales proyectos, a la fecha las iniciativas existentes aún no han alcanzado los niveles de producción necesarios para satisfacer la demanda regional y mundial.

La producción mundial de las vacunas contra la COVID-19 que cuentan con autorizaciones para su distribución y administración sigue concentrada en algunos fabricantes, los cuales, además, encuentran obstáculos para acelerar y ampliar la producción debido a las limitaciones a escala mundial en la fabricación de materiales e insumos para la producción de vacunas (por ejemplo, excipientes biológicos y viales), mientras que últimamente la OMS anunció que facilitará el establecimiento de centros de transferencia de un paquete tecnológico completo y proporcionar entrenamiento a fabricantes en países de ingresos medianos y bajos, priorizando inicialmente la tecnología de vacunas de ARNm, habiendo sido elegidos Brasil y Argentina ${ }^{(10)}$.

La necesidad de asegurar un suministro suficiente de vacunas es fundamental para asegurar el éxito de los programas de inmunización. Sin embargo, las inversiones deben cumplir con ciertas medidas de rentabilidad para ser económicamente justificadas, de tal manera que, para instalar nuevas plantas de producción de vacunas, para ser sostenible tanto la producción y los ingresos deben generar retornos que pueda justificar la inversión. Inversores, ya sean gobiernos o agencias de financiación, deben poder tener un plan claro sobre cuánto tiempo se requerirán subsidios, si los hubiere, y a qué escala. El establecimiento de la producción de vacunas generalmente comprende tres componentes: primero el establecimiento de una instalación y adquisición de equipos; seguido de procesos ascendentes, la participación de ensayos clínicos y preclínicos al inicio de una nueva producción; este proceso depende de si la tecnología de la vacuna utilizado es nuevo o reinventado a partir de productos originarios, existentes, y finalmente una vez completado, hay dos fases de fabricación: Procesos de producción y llenado de vacunas a granel ${ }^{(11)}$. Munira et al realizaron un estudio que permita calcular los costos por dosis, de establecer la producción local de vacunas en los países en desarrollo sobre la base de los datos proporcionados por productores de vacunas, encontrando que el costo por dosis promedio general de producir vacunas en los países en desarrollo fue de US $\$ 2,18$, con un rango entre US $\$ 0,98$ (para vacunas multidosis) y US $\$ 4,85$ (para nuevas vacunas). Estas estimaciones están de acuerdo con los costos reportados de las vacunas producidas por multinacionales, que sugirió costos que oscilan entre $0,05,3$ y US $\$ 4$ por dosis. La OMS informa que el $85 \%$ de la población mundial vive en países de ingresos bajos y medianos, mientras que el $93 \%$ de la carga de morbilidad se encuentra en estos países ${ }^{(11)}$.

Dentro de los Objetivos de Desarrollo Sostenible (ODS), en el $\mathrm{N}^{\circ} 3$ se menciona: «Garantizar una vida sana y promover el bienestar de todos a todas las edades», y en el que debemos resaltar el item $3 \mathrm{~b}$ que menciona «Apoyar las actividades de investigación y desarrollo de vacunas y medicamentos contra las enfermedades transmisibles y no transmisibles que afectan primordialmente a los países en desarrollo y facilitar el acceso a medicamentos y vacunas esenciales ..», objetivos que debieran implementarse hasta el 2030 y las propuestas de desarrollos tecnológicos se enmarcan en estos acuerdos multilaterales.

En todo este contexto, siendo necesario tener una respuesta oportuna y sostenible en emergencias sanitarias para cubrir las necesidades de vacunas en nuestro país considerando además el entorno internacional que viene propugnando la formación de redes y socios para la transferencia tecnológica orientadas a la producción de vacunas para países en vías de desarrollo, es la oportunidad de que el Perú cuente con una planta de vacunas, no solo contra el COVID-19 si no para estar preparados con capacidades de hacer frente a otras pandemias que puedan emerger y también nos permita producir vacunas para enfermedades desatendidas en la región como la rabia, la fiebre amarilla, el dengue y otros arbovirus, que por no tener una rentabilidad económica no es de interés de los laboratorios transnacionales, pero que para nuestro país y la región tienen una rentabilidad social que nos permita cerrar las brechas de inequidad que limitan nuestro desarrollo integral. Evidentemente es imprescindible para este propósito la transferencia tecnológica contando con la cooperación de centros de investigación y producción de la región y de otros 
países desarrollados. Hay el antecedente histórico de que el Perú contribuyó a la erradicación de la viruela produciendo la vacuna antivariólica liofilizada en la década de los 70 , en el 2013 se procuró una estrategia de transferencia tecnológica inversa con Francia para producción de la vacuna contra fiebre amarilla. Actualmente consideramos que se dan las condiciones para iniciar la implementación de una planta de producción de vacunas, estableciendo políticas públicas del corto, mediano y largo plazo, para enfrentar oportuna y sostenidamente pandemias, epidemias y controlar las endemias de enfermedades desatendidas, que además de la pérdida de vidas humanas, frenan el desarrollo integral y armónico de nuestro país.

\section{REFERENCIAS BIBLIOGRÁFICAS}

1. World Health Organization. WHO Coronavirus COVID-19. Dashboard [Internet]. Geneva: WHO; 2021 [citado el 28 setiembre de 2021]. Disponible en: https://covid19.who.int/

2. Ministerio de Salud del Perú. Sala Situacional Covid-19, Perú [Internet] Lima: MINSA; 2021 [citado el 28 setiembre de 2021]. Disponible en: https://covid19.minsa.gob.pe/sala_situacional.asp

3. Pifarré I Arolas H, Acosta E, López-Casasnovas G, Lo A, Nicodemo C, Riffe T, Myrskylä M. Years of life lost to COVID-19 in 81 countries. Sci Rep. 2021;11(1):3504. doi: 10.1038/s41598-021-83040-3.

4. Morens DM, Fauci AS. Emerging Pandemic Diseases: How We Got to COVID-19. Cell. 2020;182(5):1077-1092. doi: 10.1016/j. cell.2020.08.021.

5. Morens D.M., Daszak P., Taubenberger J.K. Escaping Pandora’s Box - Another Novel Coronavirus. N Engl J Med. 2020;382:1293-1295.

6. Saad LD, Barata RB. Yellow fever outbreaks in São Paulo State, Brazil, 2000-2010. Epidemiol Serv Saude. 2016;25(3):531-540. doi: 10.5123/ S1679-49742016000300009.

7. Organización para la Cooperación y el Desarrollo Económicos. Exploitative pricing in the time of COVID-19 [Internet]. París: OCDE; 2020 [citado el 28 setiembre de 2021]. Disponible en inglés en: https://www.oecd.org/competition/Exploitativepricing-in-thetime-of-COVID-19.pdf
El continuo desarrollo de la ciencia con certeza nos traerá nuevos medicamentos, vacunas, y técnicas de diagnóstico; sin embargo, no hay razón para pensar que estos aportes solos podrán superar la amenaza de enfermedades emergentes cada vez más frecuentes y mortales. Lo que viene ocurriendo en las últimas décadas nos induce a pensar que el SARS, MERS y COVID-19 son solo los ejemplos más recientes de un aluvión mortal del coronavirus venidero y otras emergencias para las cuales no solamente debemos estar bien preparados con todo lo aprendido en esta pandemia como país, como Región y como habitantes de esta nuestra aldea global; si no también vivir en estrecha e inteligente armonía con la naturaleza.

8. Organización Mundial del Comercio. Informe anual del Director General. Revista general de la evolución del entorno comercial internacional (documento WT/TPR/OV/23) [Internet]. Ginebra; OMC; 2020 [citado el 28 setiembre de 2021]. Disponible en: https:// docs.wto.org/dol2fe/Pages/SS/directdoc.aspx?filename=s:/WT/TPR/ OV23.pdf

9. Organización Mundial de la Salud. COVAX: colaboración para un acceso equitativo mundial a las vacunas contra la COVID-19 [Internet]. Ginebra: OMS; 2021 [citado el 28 setiembre de 2021]. Disponible en: https://www.who.int/es/initiatives/act-accelerator/ covax

10. World Health Organization. Establishment of a COVID-19 mRNA vaccine technology transfer hub to scale up global manufacturing [Internet]. Geneva: WHO; 2021 [citado el 28 setiembre de 2021] Disponible en: https://www.who.int/news-room/articles-detail/ establishment-of-acovid-19-mrna-vaccine-technology-transfer-hub-to-scale-up-global-manufacturing

11. Munira SL, Hendriks JT, Atmosukarto II, Friede MH, Carter LM, Butler JRG, Clements ACA. A cost analysis of producing vaccines in developing countries. Vaccine. 2019;37(9):1245-1251. doi: 10.1016/j. vaccine.2018.11.050. 